\title{
SEISMIC TRAVELTIME TOMOGRAPHY IN THE DOM JOÃO FIELD, RECÔNCAVO BASIN, BRAZIL
}

\author{
Naiane Pereira de Oliveira ${ }^{1}$ and Amin Bassrei ${ }^{2}$
}

\begin{abstract}
Tomography was incorporated in Exploration Geophysics with the intention of providing high-resolution images of regions in Earth's subsurface that are characterized as potential reservoirs. In this work, seismic traveltime tomography in the transmission mode was applied to real data from the Dom João Field, Recôncavo Basin, State of Bahia, Brazil. This basin represents a landmark of oil exploration in Brazil and has been intensively studied since the 1950's. Today, the Recôncavo Basin is still the principal oil producer in the State of Bahia, but there is a demand for new technologies, especially for mature fields, to improve hydrocarbon recovery. Acoustic ray tracing for the computation of traveltimes was used for forward modeling, and the conjugate gradient algorithm with regularization through derivative matrices was used as the inverse procedure. The estimated tomograms were consistent with available data from a sonic log near the acquisition area in terms of the layer geometry, as well as the P-wave velocity range. The results showed that traveltime tomography is feasible for the characterization of reservoirs with a high rate of vertical change, similar to the Dom João Field.
\end{abstract}

Keywords: traveltime tomography, seismic inversion, regularization, reservoir characterization, Recôncavo Basin.

RESUMO. A tomografia foi incorporada na Geofísica de Exploração justamente para fornecer imagens de alta resolução de regiões do interior da Terra, consideradas como potenciais reservatórios. Neste trabalho aplicamos a tomografia sísmica de tempos de trânsito no modo de transmissão em dados reais do Campo de Dom João, Bacia do Recôncavo, Estado da Bahia, Brasil. Esta bacia representa um marco da exploração de petróleo no Brasil e vem sendo exaustivamente estudada desde a década de 1950. Embora haja uma demanda por novas tecnologias, em especial para campos maduros, com o propósito de se aumentar a recuperação de hidrocarbonetos, a Bacia do Recôncavo é ainda a principal produtora do Estado da Bahia. Para o procedimento da modelagem direta foi utilizado o traçado de raios acústicos e para o procedimento inverso foi utilizado o algoritmo do gradiente conjugado com regularização através de matrizes de derivadas. Os tomogramas estimados foram consistentes com os dados provenientes do perfil sônico de um poço próximo ao levantamento tomográfico analisado, tanto em termos de geometria de camadas, como também na faixa de velocidades da onda P. Os resultados mostraram que a tomografia de tempos de trânsito é viável para a caracterização de reservatórios com elevada taxa de variação vertical, que é o caso do Campo de Dom João.

Palavras-chave: tomografia de tempos de trânsito, inversão sísmica, regularização, caracterização de reservatórios, Bacia do Recôncavo.

\footnotetext{
1 Universidade Área 1; Universidade Federal da Bahia, Instituto de Geociências, Rua Barão de Jeremoabo, s/n, Ondina 40170-115 Salvador, BA, Brazil. Phone: +55(71) 3283-8508- E-mail: naipereiraoliveira@gmail.com

2Universidade Federal da Bahia, Instituto de Geociências, Centro de Pesquisa em Geofísica e Geologia, Instituto Nacional de Ciência e Tecnologia em Geofísica do Petróleo, Rua Barão de Jeremoabo, s/n, Ondina 40170-115 Salvador, BA, Brazil. Phone: +55(71) 3283-8508 - E-mail: bassrei@ufba.br
} 


\section{INTRODUCTION}

Tomography is an established technique in medicine and has been used in Exploration Seismology to create high-resolution images of the Earth's subsurface of potential reservoirs or already confirmed reservoirs under development. Tomography is a special type of inverse problem that estimates a function using integrals along lines.

In Exploration Seismology, the tomographic technique is grouped into two classes: traveltime tomography, which takes into account only the traveltimes between the sources and the receivers, and dynamic tomography, which also considers the waveform of the signal received by the receiver. The tomography classification is also determined by the wave used in the survey, that is, a direct, reflected or refracted wave. There are also tomography formulations that use more than one type of wave.

In recent years, seismic tomography has been used for new applications, such as more accurate estimations of velocity macro models for seismic migration. This is the case for stereotomography, which estimates velocity models from seismic reflection data and the local inclination associated with picking events.

In this work, we used a kinematic approach of transmission tomography for which the input data were the vector of observed traveltimes between the sources and the receivers and the model parameter to be estimated was the slowness (reciprocal of velocity) distribution of the 2-D medium. Piezoelectric sources were used to generate ultrasonic pulses whose propagation path may be modeled by the kinematic ray theory, which is a high frequency approximation of the sound propagation phenomenon.

The Recôncavo Basin, in the State of Bahia, Brazil, has a great historical and economic importance. After many years of production, this basin is now in an advanced exploration stage. Motivated by the effective oil recovery in mature fields, highly advanced technology has been used in this basin to characterize its mature fields. For this purpose, seismic tomographic surveys have been conducted by PETROBRAS in some oil fields, such as the Dom João Field.

The tomographic inversion is an ill-posed problem because the existence, uniqueness and stability conditions are not completely satisfied. Mathematical techniques are used with the intention of providing better conditioning for the numerical solution of such problems. Iterative algorithms with regularization to solve linear systems are committed to provide reliable solutions. The input data in the system are the traveltimes between the sources and the receivers and the distances propagated by each ray connecting such sources and receivers. Both inputs are provided through acoustic forward modeling. The linear system is regularized by derivative matrices derived to minimize the instability.
This regularization procedure has a crucially important parameter, $\lambda$. We chose the so-called L-module proposed by Sá (1996) for $\lambda$. The idea of the $L$-module was based on the well-known L-curve.

\section{Inverse Problems, Linearization, Regularization and L-module}

Inversion is a technique that estimates the parameters of a given model using the observed data as an input. On the other hand, forward modeling provides such data by assuming a synthetic model. While the output of the forward problem is theoretically unique, inverse problems show an inherent ambiguity in the conclusions that can be obtained.

The information description is used as the starting point when analyzing geophysical data. A vector provides a practical representation of these values: $\boldsymbol{d}=\left[d_{1}, d_{2}, \ldots, d_{M}\right]^{T}$ is the observed data parameters or simply observed data. The model parameters, or simple model, is represented by $\boldsymbol{m}=$ $\left[m_{1}, m_{2}, \ldots, m_{N}\right]^{T}$. For the linear problem, the equation $\boldsymbol{d}=\boldsymbol{G} \boldsymbol{m}$ represents the solution of the forward problem, and $\boldsymbol{m}^{\text {est }}=\boldsymbol{G}^{-1} \boldsymbol{d}$ represents the solution of the inverse problem. To solve an inverse problem, we must analyze the existing issues, uniqueness and stability. If one of these conditions fail, the problem is said to be ill-posed.

The method of linearization starts from an initial model, $\boldsymbol{m}_{o}$, which is updated successively and is also known as the GaussNewton method. It linearizes the nonlinear problem $\boldsymbol{g}(\boldsymbol{m})=\boldsymbol{d}$ around an approximate solution. The estimated model is then obtained iteratively by solving a system of linear equations at each step. This method will converge to the model associated with the cost function closer to $S\left(\boldsymbol{m}_{o}\right)$ (Menke, 1989).

The expression to update the current model is:

$$
\left(\boldsymbol{G}^{T}\right)^{k} \Delta \boldsymbol{d}^{k}=\left(\boldsymbol{G}^{T} \boldsymbol{G}\right)^{\boldsymbol{k}} \Delta \boldsymbol{m}^{k},
$$

where $\Delta \boldsymbol{m}^{k}=\boldsymbol{m}^{k+1}-\boldsymbol{m}^{k}$ is the estimated model parameters update for $k$-th iteration, $(\boldsymbol{G})^{k}$ is the tomographic matrix for the $k$-th iteration, and $\Delta \boldsymbol{d}^{k}$ is the data residual between the $k+1$-th and the $k$-th iterations.

Regularization techniques are applied in the numerical solution of discrete ill-posed problems to make the inversion more stable. These techniques do not solve the original problem, but an equivalent one, which is more robust in relation to the variations in the input data. The regularization by derivative matrices is expressed by the objective function $\varphi(\boldsymbol{m})$ (Bassrei \& Rodi, 1993):

$$
\varphi(\boldsymbol{m})=\boldsymbol{e}^{T} \boldsymbol{e}+\lambda L_{n},
$$


where $L_{n}$ is a discrete derivative operator that imposes some smoothing to the solution and $\lambda$ is a non-negative constant called the regularization parameter, which controls the amount of smoothing on the solution. We can use the difference between the physically adjacent model parameters as an approximation of the first derivative $(n=1)$ matrix expressed as $\boldsymbol{D}_{1}$. The operator, also known as flatness, will then be $L_{1}=\boldsymbol{m}^{T} \boldsymbol{D}_{1}^{T} \boldsymbol{D}_{1} \boldsymbol{m}$ (Menke, 1989). We can also use a matrix that approximates the second derivative $(n=2)$, expressed as $\boldsymbol{D}_{2}$. The operator, $L_{2}=\boldsymbol{m}^{T} \boldsymbol{D}_{2}^{T} \boldsymbol{D}_{2} \boldsymbol{m}$, is called roughness (Menke, 1989). The general expression for $L_{n}$, either flatness or roughness is given by:

$$
L_{n}=\left\|l_{n}\right\|_{2}^{2}=\left(\boldsymbol{D}_{n} \boldsymbol{m}\right)^{T} \boldsymbol{D}_{n} \boldsymbol{m},
$$

where $n$ is the order of the derivative matrix. By developing the equation $\varphi(\boldsymbol{m})$ and using the appropriate substitutions $\boldsymbol{e}=\boldsymbol{d}-\boldsymbol{G} \boldsymbol{m}$ and $L_{n}=\left(\boldsymbol{D}_{n} \boldsymbol{m}\right)^{T} \boldsymbol{D}_{n} \boldsymbol{m}$, we have:

$$
\begin{aligned}
\varphi(\boldsymbol{m})= & \left(\boldsymbol{d}^{T}-\boldsymbol{m}^{T} \boldsymbol{G}^{T}\right)(\boldsymbol{d}-\boldsymbol{G} \boldsymbol{m}) \\
& +\boldsymbol{\lambda}\left(\boldsymbol{D}_{n} \boldsymbol{m}\right)^{T} \boldsymbol{D}_{n} \boldsymbol{m} .
\end{aligned}
$$

When $\lambda=0$, there is no regularization and the inversion is reduced to the standard least squares method. Minimizing this objective function $\varphi(\boldsymbol{m})$ in relation to the model parameters $\boldsymbol{m}$ and equating to zero, we obtain the following system of linear equations:

$$
\left(\boldsymbol{G}^{T} \boldsymbol{G}+\boldsymbol{\lambda} \boldsymbol{D}_{n}^{T} \boldsymbol{D}_{n}\right) \boldsymbol{m}^{k}=\boldsymbol{G}^{T} \boldsymbol{d}^{k} .
$$

When $n=0, \boldsymbol{D}_{n}$ corresponds to the identity matrix resulting in a zero-order regularization, and the solution reduces to the damped least squares method. When $n=1, \boldsymbol{D}_{n}$ corresponds to the first derivative matrix, and the regularization is then called first-order. When $n=2$, we have the second-order regularization, which uses the numerical approximation of the second derivative.

For the linearized process, we started with a homogeneous initial model $\boldsymbol{m}^{0}$ and updated the model parameters iteratively through the equation:

$$
\left(\boldsymbol{G}^{T} \boldsymbol{G}+\boldsymbol{\lambda} \boldsymbol{D}_{n}^{T} \boldsymbol{D}_{n}\right)^{k} \Delta \boldsymbol{m}^{k}=\left(\boldsymbol{G}^{T}\right)^{k} \Delta \boldsymbol{d}^{k} .
$$

There are several methods presented in the literature to find the optimal regularization parameter $\lambda$. Among them, the $\mathrm{L}-$ module, which was proposed by Sá (1996), is based on the Lcurve. The concept of the L-curve is necessary to understand the L-module. The L-curve is a graphical tool used to choose the regularization parameter. Some recent applications of the L-curve in Exploration Geophysics can be seen in Santos et al. (2006, 2009).
The abscissas correspond to the error vector between the observed and calculated data, $\|\boldsymbol{e}\|=\|\boldsymbol{G} \boldsymbol{m}-\boldsymbol{d}\|$. The ordinates correspond to the amount of regularization, $\left\|\boldsymbol{D}_{n} \boldsymbol{m}\right\|$. When displayed on log-log scale, this curve usually resembles the shape of the letter 'L', where the inflection region represents the region of interest because it indicates the best regularization parameter. Thus, each point on this curve is the result of an estimated model by which its turn is related to $\lambda$, and the ideal point expresses the balance between the error and the regularization.

The L-module measures the distance of a point on the Lcurve to the axes origin and is defined by the following equation (Sá, 1996):

$$
M O D L^{2}=\left[\boldsymbol{e}^{T} \boldsymbol{e}\right]^{2}+\left[\left(\boldsymbol{D}_{n} \boldsymbol{m}\right)^{T}\left(\boldsymbol{D}_{n} \boldsymbol{m}\right)\right]^{2}
$$

It is not necessary to construct the L-curve, and the interest region minimizes the $\mathrm{L}$-module. This method has another version if the input data are corrupted by noise, according to the following formula (Sá, 1996):

$$
\begin{aligned}
M O D L^{2}= & {\left[\boldsymbol{e}^{T} \boldsymbol{e}-\boldsymbol{r}^{T} \boldsymbol{r}\right]^{2} } \\
& +\left[\left(\boldsymbol{D}_{n} \boldsymbol{m}\right)^{T}\left(\boldsymbol{D}_{n} \boldsymbol{m}\right)\right]^{2}
\end{aligned}
$$

where $\boldsymbol{r}$ is the vector that represents the noise in the data. When the noise level added in the data is unknown, an estimate may be used. Again, the curve must have a minimum.

\section{Seismic Tomography in Dom João Field}

Discovered in 1947, the Dom João Field is located in the Recôncavo Basin. This basin is located in the state of Bahia, northeastern Brazil, whose boundaries are the Aporá High to the north and northwest, the Barra fault system to the south, the Maragogipe fault to the west, and the Salvador fault system to the east. It occupies an area of approximately $11,500 \mathrm{~km}^{2}$ according to Silva et al. (2007). It has a historical importance for Petroleum Geology in Brazil because it was the first producing basin. It has been intensively studied over the past five decades by PETROBRAS, which has resulted in approximately 5,050 drilled wells and 30,000 km of recorded reflection seismic sections (Ribeiro \& Borghi, 2003).

The oil accumulation of the Dom João Field is concentrated mainly in the sandstones of the Sergi Formation and secondarily in the Água Grande Formation. The field has an area of $47 \mathrm{~km}^{2}$, two-thirds of which are under water in the Todos os Santos Bay (Milani \& Araújo, 2003). The field structure is an elongated horst in the SSW-NNE direction and is approximately $24 \mathrm{~km}$ long with normal faults that limit this structural feature: Dom João fault to the east and Nova América fault to the west. Also, according to Milani \& Araújo (2003), the production areas of the Sergi 
Formation occur at depths of approximately 200 to $300 \mathrm{~m}$. The Candeias-Sergi-Ilhas petroleum system has lacustrine shale as source rock from the Candeias Formation, where normal faults and blocks rotated juxtapose laterally the source rock and the reservoir, allowing the oil to directly migrate into the structural high of the basin.

The sandstones of the Sergi Formation are reservoirs of greater extension and importance of the Recôncavo Basin, with an original oil in place volume of 362 million $\mathrm{km}^{3}$ (Scherer \& Ros, 2009). The reservoirs range from fine sandstones to very gross conglomerates that accumulated during the Late Jurassic. The sandstones are present throughout the basin and have a maximum thickness of $450 \mathrm{~m}$. According to Scherer and Ros (2009), fluvio-aeolian deposits like the ones from the Sergi Formation show a very complex depositional history that generates significantly heterogeneities in the reservoirs. In addition to depositional differences, there are systematic differences in the dominant diagenetic processes. As a result, the Sergi deposits present fast variation rates in lateral and vertical petrophysical characteristics, decisively influencing the hydrocarbon recovery.

Preceding the processing of real data, we performed simulations on synthetic data inspired by the same real data. This procedure assisted us in evaluating the ray tracing program. Figure 1 shows the geologic model that has a velocity range from 2800 to $4000 \mathrm{~m} / \mathrm{s}$. The model has 43 blocks in the horizontal direction and 82 blocks in the vertical direction, totaling 3,526 blocks. The velocity (or slowness) is constant in each square block with dimensions of $5 \times 5 \mathrm{~m}$. In terms of data acquisition geometry, one well contains 141 sources and the other contains 140 receivers, so there are 19,740 traveltimes. The linear system is overdetermined with 19,740 data parameters or equations and 3,526 model parameters or unknowns.

Due to the size of the problem, the matrix inversion approach would be too costly. We used the conjugate gradient method inserted into the linearized inversion scheme. The parameters used in the simulations with synthetic data came from the field data. Thus, the source and the receiver depths from the field acquisition were used in the ray tracing algorithm. Our initial velocity model corresponded to a homogeneous medium: a sandstone with the P-wave velocity equal to $3,100 \mathrm{~m} / \mathrm{s}$. The initial guess is very important, because according to Menke (1989), the linearized method does not "see" the entire error surface $S(\boldsymbol{m})$. Instead, it sees only the part of $S(\boldsymbol{m})$ in the vicinity of $\boldsymbol{m}_{o}$ and approximates the rest of the surface as a paraboloid tangent to the actual surface at that point. The new estimate is the minimum of that paraboloid. Since any minimum with continuous derivates is locally paraboloid in shape, the method will converge to the minimum of the error function if the initial guess is close enough.

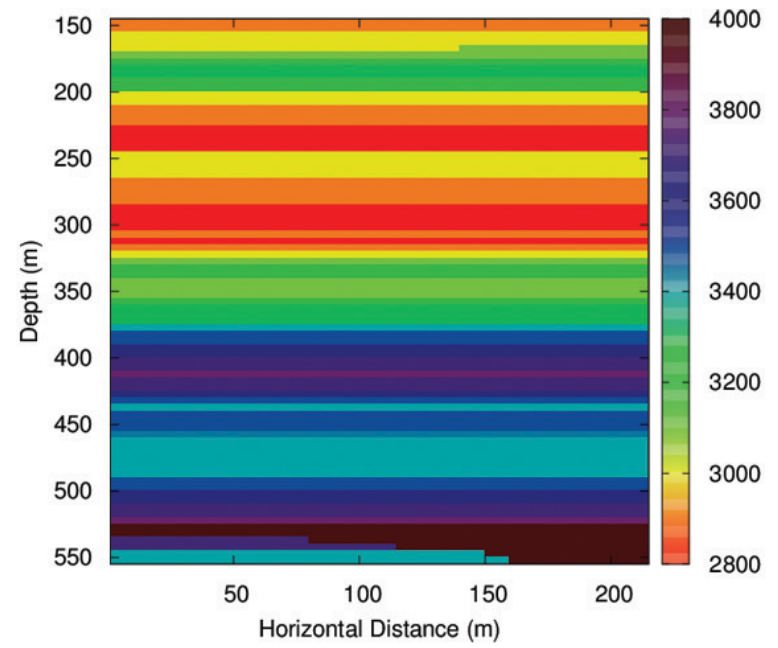

Figure 1 - True model for seismic tomography, suggested from real data from the Dom João Field, Recôncavo Basin. The color bar indicates the P-wave velocities in $\mathrm{m} / \mathrm{s}$.

Using the initial velocity distribution, the rays were computed and the traveltimes were calculated. Each calculated traveltime was compared to the observed traveltime, so the velocity model was updated for each inversion iteration. Thus, the first ray trace was with straight rays, and the following one was with curved rays because the velocity model was updated for each iteration. The processing time for each iteration was approximately $45 \mathrm{~min}$ utes using a PC with the Intel ${ }^{\circledR}$ Core $^{\mathrm{TM}}$ processor with $2.50 \mathrm{GHz}$ and 6 GBytes RAM memory. We used the curved ray tomography approach, where the non-linear inversion was linearized through Taylor's series, and we tested 20 different regularization parameters $\lambda$ for each iteration.

In order to make the simulations closer to the field conditions, Gaussian noise was added to the traveltime data. To select the regularization parameter $\lambda$, we used (i) the L-module approach (Figs. 2a and 3a), (ii) the curve of the Root Mean Square (RMS) traveltime error $E_{d, r m s}$ (Figs. 2b and $3 \mathrm{~b}$ ), and (iii) the curve of the RMS velocity error $E_{v, r m s}$ (Figs. 2c and 3c). Figures $2 \mathrm{a}, 2 \mathrm{~b}$ and $2 \mathrm{c}$ are related to the first-order regularization, and Figures $3 a, 3 b$ and $3 c$ are related to the second-order regularization. The first-order regularization convergence was achieved with four iterations. Figure 4a shows the result after the first iteration, and Figure $4 b$ shows the result after the fourth one. For the second-order regularization, Figure 5a shows the estimated tomogram after the first iteration, and Figure $5 b$ shows the estimated tomogram after the fourth one. The estimated tomograms were consistent with the true model in terms of layer geometry, 
as well as the P-wave velocity range. The RMS error between the true velocities and the estimated velocity was approximately 1.80 to $3.48 \%$, depending on the regularization order and the iteration.

(a)

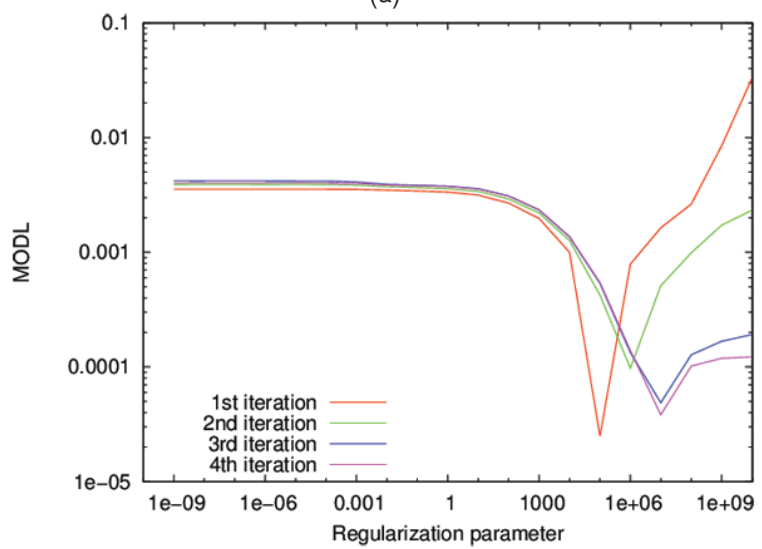

(b)

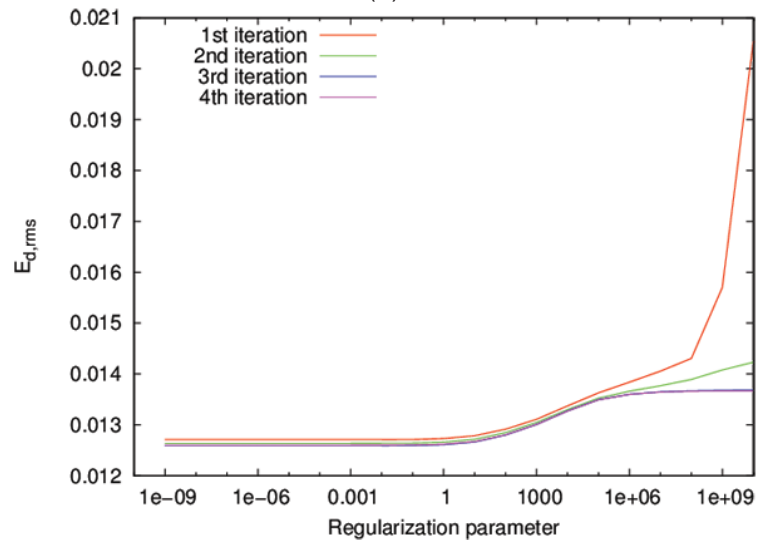

(c)

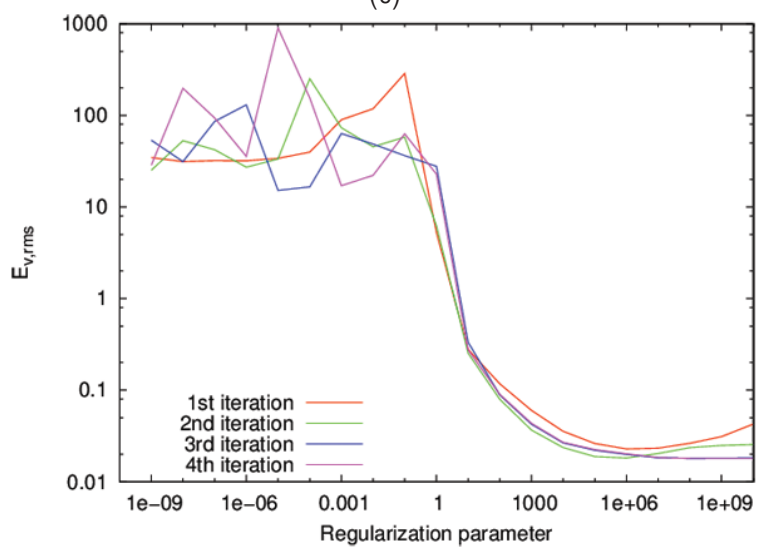

Figure 2 - Auxiliary curves for the synthetic data corresponding to different iterations used to choose the optimal regularization parameter using the first-order regularization. The linearized inversion was done with the conjugate gradient, and the data were corrupted with random noise (noise factor $\mu=0.1$ ): (a) MODL curve; (b) RMS error curve between the observed traveltimes and the calculated traveltimes; (c) RMS error curve between the true velocities and the estimated velocities. (a)



(b)



(c)

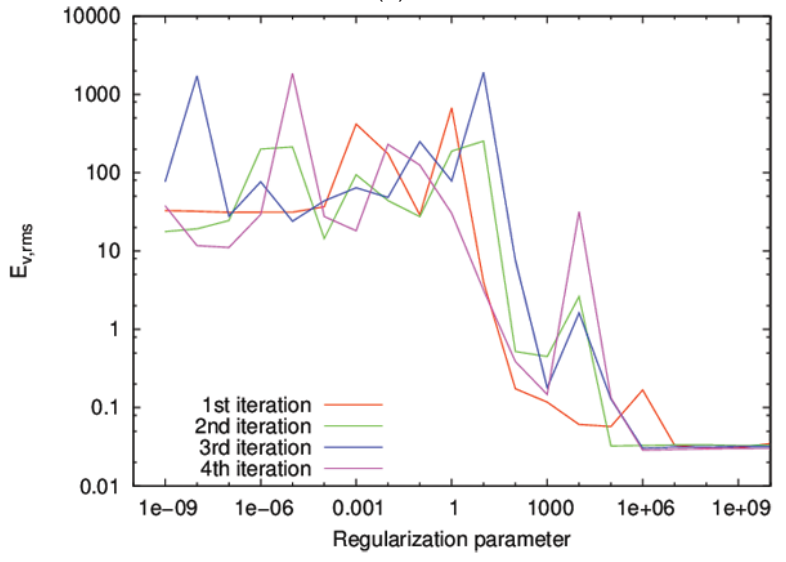

Figure 3 - Auxiliary curves for the synthetic data corresponding to different iterations used to choose the optimal regularization parameter using the second-order regularization. The linearized inversion was done with the conjugate gradient, and the data were corrupted with random noise (noise factor $\mu=0.1$ ): (a) MODL curve; (b) RMS error curve between the observed traveltimes and the calculated traveltimes; (c) RMS error curve between the true velocities and the estimated velocities.

Table 1 summarizes all of the simulations with synthetic data using a linearized inversion through the conjugate gradient 
method with regularization by derivative matrices. The data were corrupted with random noise (noise factor $\mu=0.1$ ). Both regularization orders were used, i.e., first-order $n=1$ and secondorder $n=2$. The optimum regularization parameter was selected using the L-module criterion (Figs. 2a and 3a). The stop criterion was the RMS residual between the estimated velocity distribution between iterations $k$ and $k+1$ :

$$
\Delta E_{v, r m s}=1 / N \sqrt{\sum_{i=1}^{N}\left(v_{i}^{k+1}-v_{i}^{k}\right)^{2}} .
$$

Table 1 - Simulation results with synthetic data using a linearized inversion through the conjugate gradient method with a regularization by derivative matrices. The data were corrupted with random noise (noise factor $\mu=0.1$ ). $n=1$ indicates a first-order regularization, and $n=2$ indicates a second-order regularization. $\lambda^{o p t}$ is the optimum regularization parameter, and $\Delta \boldsymbol{E}_{r m s}$ is the RMS velocity residual between iterations $k+1$ and $k$.

\begin{tabular}{|c|c|c|c|c|}
\hline Iteration & 1 & 2 & 3 & 4 \\
\hline $\mathrm{n}$ & 1 & 1 & 1 & 1 \\
$\lambda^{o p t}$ & $10^{8}$ & $10^{6}$ & $10^{8}$ & $10^{8}$ \\
$E_{d, r m s}(\%)$ & 1.43 & 1.37 & 1.37 & 1.37 \\
$E_{v, r m s}(\%)$ & 2.63 & 1.82 & 1.80 & 1.80 \\
$\Delta E_{r m s}(\mathrm{~m} / \mathrm{s})$ & 6.42 & 1.01 & 0.13 & 0.05 \\
\hline \hline $\mathrm{n}$ & 2 & 2 & 2 & 2 \\
$\lambda^{o p t}$ & $10^{10}$ & $10^{5}$ & $10^{6}$ & $10^{9}$ \\
$E_{d, r m s}(\%)$ & 1.43 & 1.35 & 1.35 & 1.35 \\
$E_{v, r m s}(\%)$ & 3.48 & 3.24 & 3.03 & 3.01 \\
$\Delta E_{r m s}(\mathrm{~m} / \mathrm{s})$ & 6.09 & 1.52 & 0.62 & 0.10 \\
\hline
\end{tabular}

A velocity residual of $0.1 \mathrm{~m} / \mathrm{s}$ or a maximum of 10 iterations was used.

The tomographic data with the crosswell geometry were acquired by Schlumberger in a research project sponsored by PETROBRAS. The single source could move in the well so that there was a total of 141 source points. Additionally, a cable with 20 receivers with a $3 \mathrm{~m}$ spacing could move in the other well, so there was a total of 140 receiver points. The wells have a certain inclination with respect to the datum plane, which was $100 \mathrm{~m}$ above sea level. Figure 6 shows the valid traveltimes as a combination of source number and receiver number. We can see that the region with deeper sources and receivers is better illumined.

Careful attention was necessary during the ray tracing procedure. For the predicted 19,740 rays numerically generated, only 14,200 were recorded in the field. We had 14,048 rays after the removal of rays with picking errors. The imaging algorithm requires the coordinates of sources and receivers, as well as a velocity model. The initial model was again homogeneous $(3,100 \mathrm{~m} / \mathrm{s})$.
The discrete model used had 3,526 blocks, with each block measuring $5 \times 5 \mathrm{~m}$. The processing time for each iteration was $40 \mathrm{~min}-$ utes, and in each iteration, the linear system is solved 20 times, each time for a different regularization parameter. Note that the inversion with real data was faster because there were less equations, although the number of unknowns was the same.

(a)



(b)

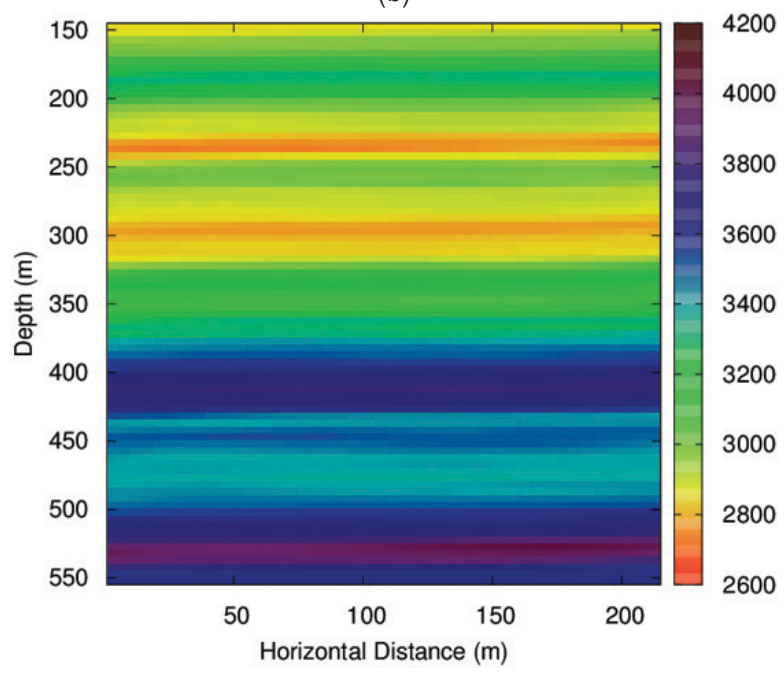

Figure 4 - Results from the synthetic data using a linearized inversion through the conjugate gradient method with a first-order regularization. The data were corrupted with random noise (noise factor $\mu=0.1$ ). (a) Estimated tomogram after the first iteration; (b) Estimated tomogram after the fourth iteration.

Once more, we used the L-module estimator to choose $\lambda$ for the first- and second-order regularization. The curves in Figures $7 \mathrm{a}$ and $8 \mathrm{a}$ show this criterion. Depending on the iteration $k$, we can estimate that the best $\lambda$ ranges from $10^{8}$ to $10^{10}$ for both orders. The first-order is shown in Figure $7 \mathrm{a}$, and secondorder is shown in Figure 8a. 
(a)



(b)

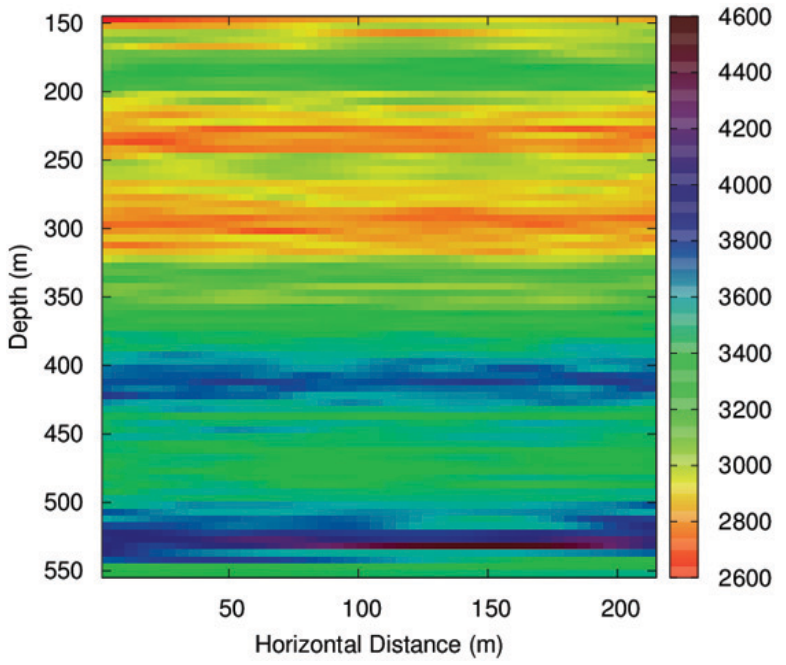

Figure 5 - Results from the synthetic data using a linearized inversion through the conjugate gradient method with a second-order regularization. The data were corrupted with random noise (noise factor $\mu=0.1$ ). (a) Estimated tomogram after the first iteration; (b) Estimated tomogram after the fourth iteration.

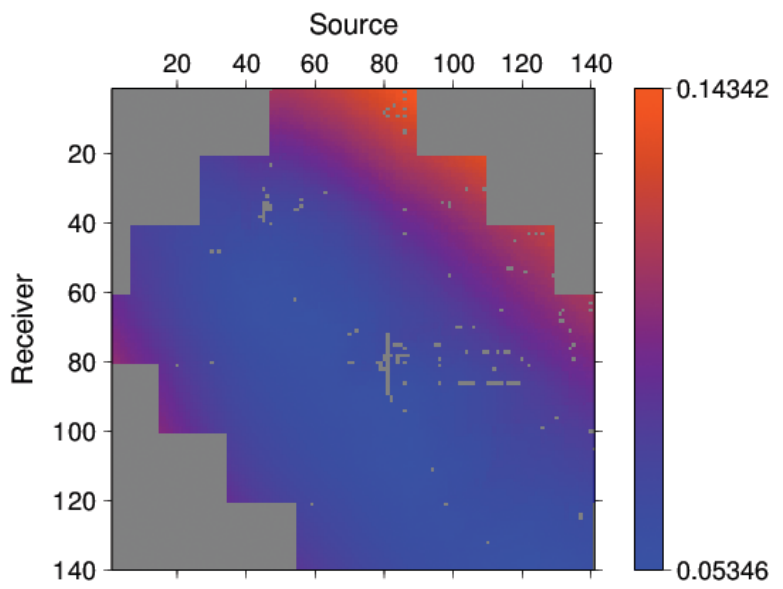

Figure 6 - Graphical representation of the traveltime from the Dom João Field. Time in seconds.

The first-order regularization convergence was achieved with seven iterations. Figure 9a shows the result after the first iteration, and Figure 9b shows the result after the seventh one. Figure 10a shows the estimated tomogram after the first iteration for the second-order regularization, and Figure 10b shows the estimated tomogram after the ninth one. The estimated tomograms were consistent with available data from a sonic log near the acquisition area in terms of layer geometry, as well as the P-wave velocity range.

Table 2 summarizes all of the simulations with real data using a linearized inversion through the conjugate gradient method with regularization by derivative matrices. Both regularization orders were used (first-order $n=1$ and second-order $n=2$ ). Simi- lar to the synthetic data, the stop criterion was the RMS residual between the estimated velocity distribution between the iterations $k$ and $k+1$. Again, a velocity residual of $0.1 \mathrm{~m} / \mathrm{s}$ or a maximum of 10 iterations was used.

\section{CONCLUSIONS}

Seismic tomography combines two important aspects: the estimation of physical properties and medium imaging. The basic idea of non-linear traveltime tomography is quite comprehensive and widely applicable in reservoir geophysics. However, it requires mathematical treatment to deal with the non-linearity and the fact the inverse problem is ill-posed. In this sense, we used the linearized inversion through the conjugate gradient method with regularization by derivative matrices. The L-module approach was used to select the optimal regularization parameter, $\lambda^{\text {opt }}$.

There is no general method for determining whether a solution obtained by the iterative method really does minimize error in the global sense. However, we believe that there is a good convergence in our case, because the studied area is reasonably well known, that is, some information about its lithology is available in the literature. This allowed us to choose an initial guess that represented satisfactorily the region. That also can be confirmed by our numerical results and the correlation with sonic profile near the acquisition area. This third well containing the sonic log is approximately $530 \mathrm{~m}$ away from the first well and $430 \mathrm{~m}$ away from the second well, being the first and the second wells the ones used in the tomographic inversion. Despite the distance, significant 
(a)

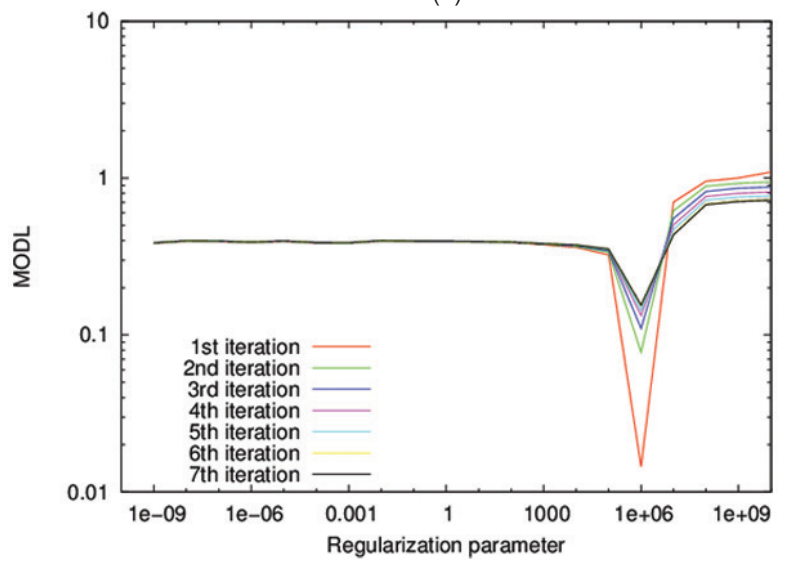

(b)

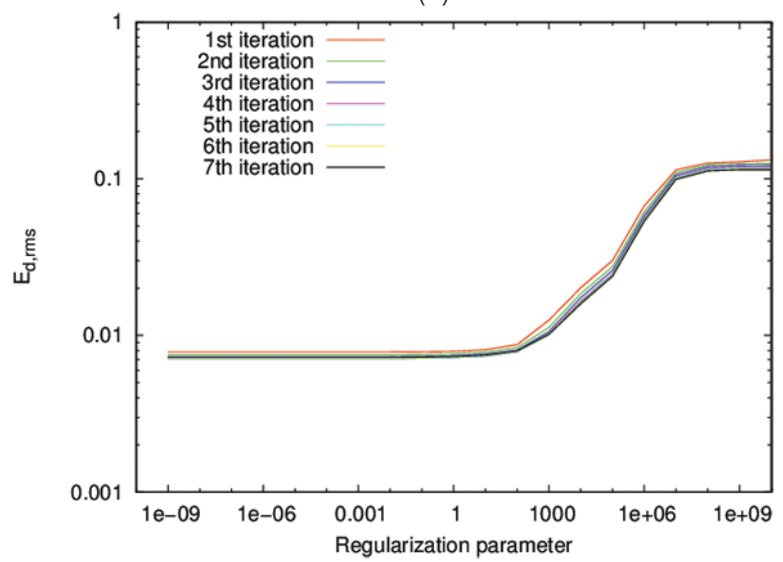

Figure 7 - Auxiliary curves for the real data corresponding to different iterations used to choose the optimal regularization parameter using a first-order regularization. The linearized inversion was done with the conjugate gradient: (a) MODL curve; (b) RMS error curve between the observed traveltimes and the calculated traveltimes.

(a)



(b)

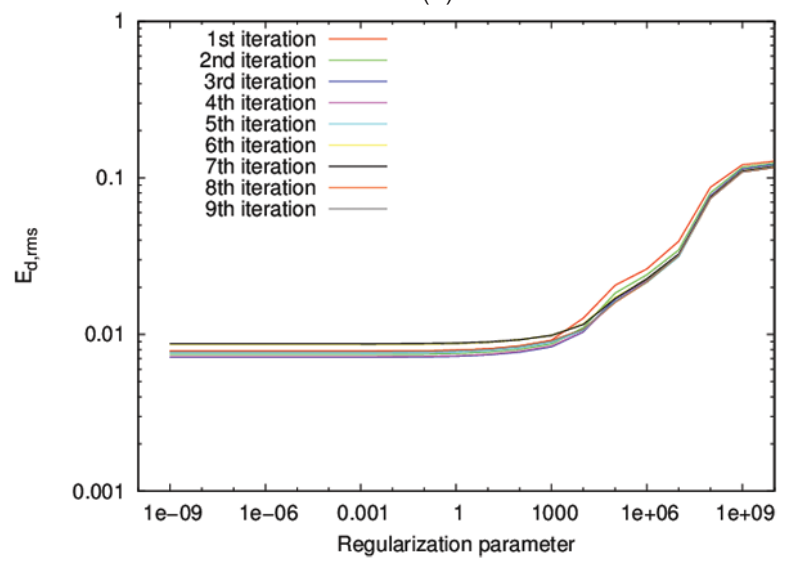

Figure 8 - Auxiliary curves for the real data corresponding to different iterations used to choose the optimal regularization parameter using a second-order regularization. The linearized inversion was done with the conjugate gradient: (a) MODL curve; (b) RMS error curve between the observed traveltimes and the calculated traveltimes.

Table 2 - Simulation results with real data using a linearized inversion through the conjugate gradient method with a regularization by derivative matrices. $n=1$ indicates a first-order regularization, and $n=2$ indicates a second-order regularization. $\lambda^{o p t}$ is the optimum regularization parameter, and $\Delta \boldsymbol{E}_{r m s}$ is the RMS velocity residual between iterations $k+1$ and $k$.

\begin{tabular}{|c|c|c|c|c|c|c|c|c|c|}
\hline Iteration & 1 & 2 & 3 & 4 & 5 & 6 & 7 & 8 & 9 \\
\hline $\mathrm{n}$ & 1 & 1 & 1 & 1 & 1 & 1 & 1 & 1 & 1 \\
$\lambda^{\text {opt }}$ & $10^{8}$ & $10^{8}$ & $10^{8}$ & $10^{8}$ & $10^{8}$ & $10^{9}$ & $10^{10}$ & - & - \\
$E_{d, r m s}(\%)$ & 12.60 & 12.28 & 11.96 & 11.67 & 11.46 & 11.44 & 11.43 & - & - \\
$E_{v, r m s}(\%)$ & 8.90 & 1.82 & 1.85 & 1.48 & 1.19 & 0.17 & 0.02 & - & - \\
\hline \hline $\mathrm{n}$ & 2 & 2 & 2 & 2 & 2 & 2 & 2 & 2 & 2 \\
$\lambda^{\text {opt }}$ & $10^{10}$ & $10^{10}$ & $10^{10}$ & $10^{10}$ & $10^{10}$ & $10^{10}$ & $10^{10}$ & $10^{10}$ & $10^{10}$ \\
$E_{d, r m s}(\%)$ & 12.76 & 12.41 & 12.13 & 11.98 & 11.88 & 11.85 & 11.78 & 11.63 & 11.63 \\
$E_{v, r m s}(\%)$ & 7.54 & 2.95 & 2.35 & 1.89 & 1.68 & 1.63 & 1.19 & 0.32 & 0.10 \\
\hline
\end{tabular}


(a)

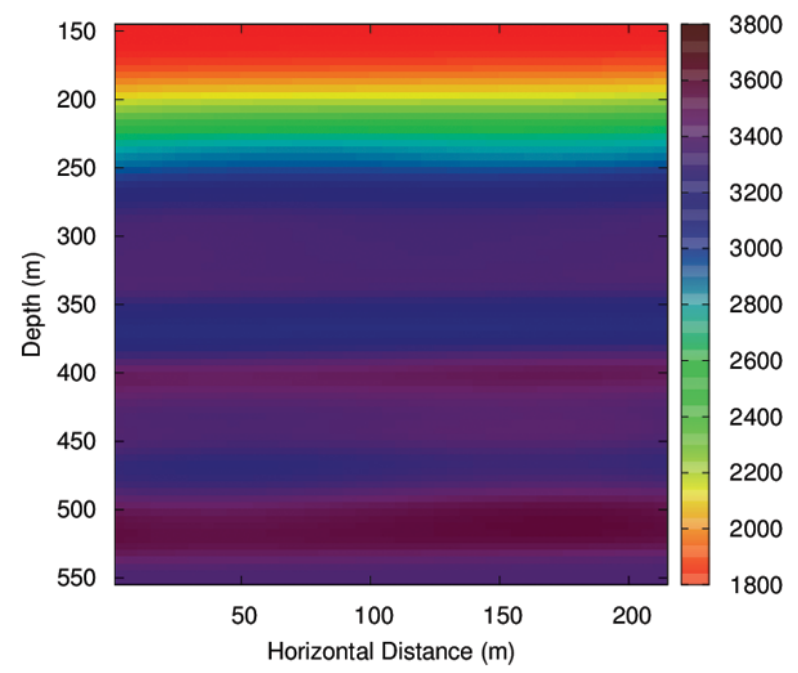

(b)

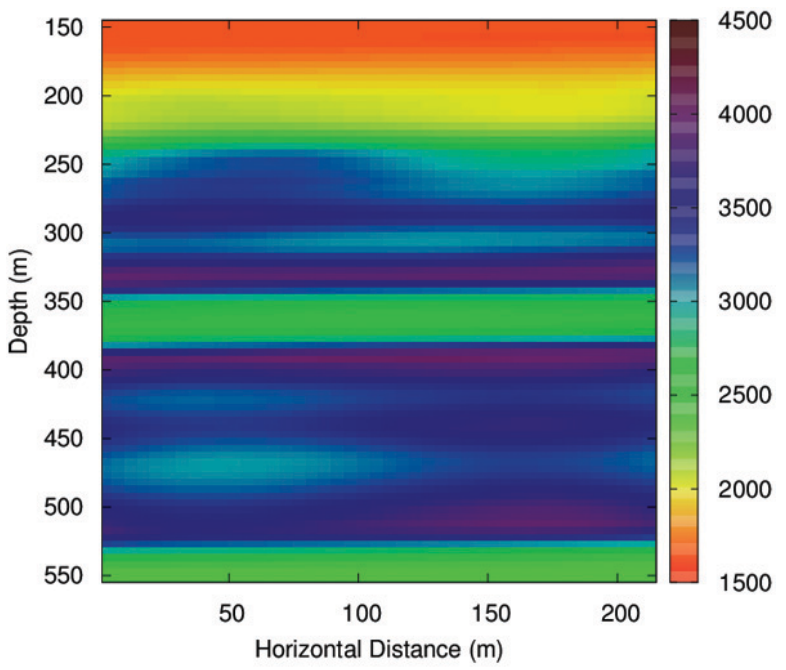

Figure 9 - Results from the synthetic data using a linearized inversion through the conjugate gradient method with a second-order regularization. The data were corrupted with random noise (noise factor $\mu=0.1$ ). (a) Estimated tomogram after the first iteration; (b) Estimated tomogram after the fourth iteration.

(a)

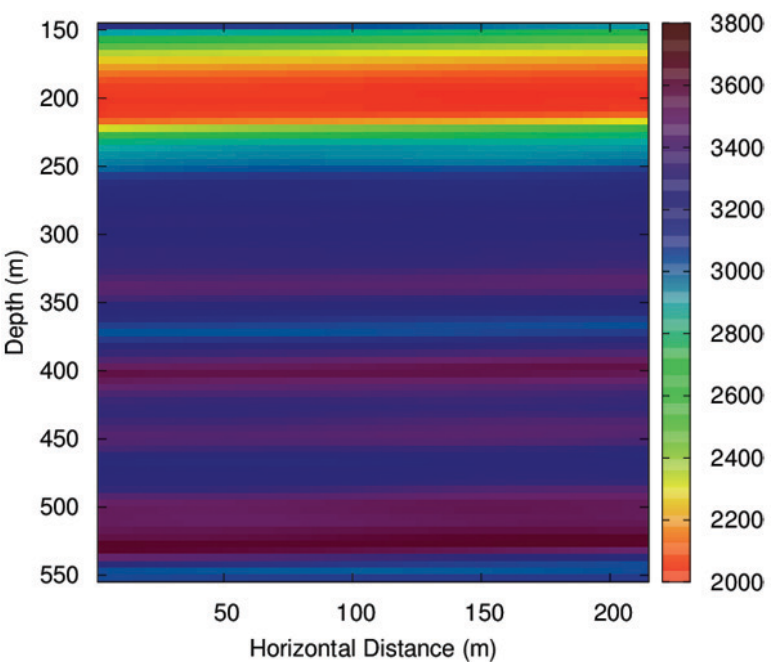

(b)

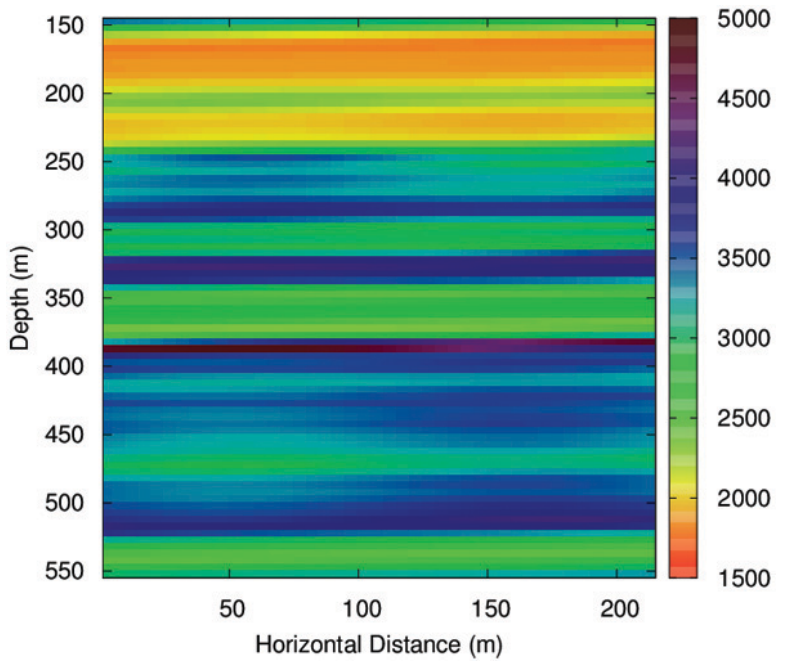

Figure 10 - Results with real data using a linearized inversion through the conjugate gradient method with a second-order regularization. (a) Estimated tomogram after the first iteration; (b) Estimated tomogram after the ninth iteration.

correlations were found between the sonic log with the estimated tomogram. A comparative analysis was performed every $50 \mathrm{~m}$ in the depth ranging between 400 and $550 \mathrm{~m}$, and the values for the estimated $\mathrm{P}$-wave velocity were consistent in most intervals.

The use of ray theory as a high-frequency approximation for acoustic wave propagation was feasible because the media in both synthetic and real data examples did not have abrupt velocity changes. There was a region at shallow depths, without rays, where the inversion result was inconsistent. One possibility to improve the quality of the solution could be to use a sonic log as a priori information because the initial model has an important role in the inversion. However, such information was not available.

In general, the results showed that traveltime tomography can be used for the characterization of reservoirs with a high rate of vertical change like the situation of the Dom João Field, Recôncavo Basin. 


\section{ACKNOWLEDGMENTS}

The authors thank PETROBRAS for sponsoring the project "Investigation on the use of Crosswell Tomography as a Toll for Complex Reservoir Characterization" and for the permission to use the real data in this work. Naiane Oliveira thanks CAPES for a M.Sc. scholarship. Amin Bassrei would like to thank CNPq for the projects, National Institute of Science and Technology in Petroleum Geophysics (INCT-GP) and 307427/2010-2 and 308690/2013-3 (research fellowship). The second author also would like to thank FINEP for sponsoring the CT-PETRO Research Network in Exploration Geophysics (Rede 01).

\section{REFERENCES}

ANDERSEN AH \& KAK AC. 1982. Digital ray tracing in two-dimensional refractive Fields. Journal of Acoustical Society of America, 72(5): 15931606.

BASSREI A \& RODI W. 1993. Regularization and inversion of linear geophysical data. In: International Congress of the Brazilian Geophysical Society, 3., SBGf: Rio de Janeiro, Brazil, v. 1, 111-116.

HESTENES MR \& STIEFEL E. 1952. Methods of Conjugate Gradients for Solving Linear Systems. Journal of Research of the National Bureau of Standards, 49(6): 409-436.

MENKE W. 1989. Geophysical Data Analysis: Discrete Inverse Theory. Academic Press, San Diego, CA. 289 pp.

MILANI EJ \& ARAÚJO LM. 2003. Recursos Minerais Energéticos: Petróleo, Geologia, Tectônica e Recursos Minerais do Brasil. CPRM, Brasilia, Brazil, pp. 541-576.

RIBEIRO MT \& BORGHI L. 2003. Depositional architecture of fluvialeolian systems: the Sergi formation, Recôncavo Basin, Brazil. In: Latin American Congress of Sedimentology, Belém, Brazil. pp. 140-142.

SÁ TJM. 1996. Inversão e Seleção de Imagens na Tomografia de Transmissão utilizando Regularização de Ordem Arbitrária, Decomposição em Valores Singulares, Conjugado Gradiente Modificado e Entropia. Master dissertation, Universidade Federal da Bahia, Salvador, Brazil. 108 pp.

SANTOS ETF, BASSREI A \& COSTA JC. 2006. Evaluation of L-Curve and Theta-Curve approaches for the selection of regularization parameters in anisotropic traveltime tomography. Journal of Seismic Exploration, 15: 245-272.

SANTOS ETF, HARRIS JM, BASSREI A \& COSTA JC. 2009. Trigonal meshes in diffraction tomography with optimum regularization: an application for carbon sequestration monitoring. Journal of Seismic Exploration, 18: 135-156.

SCHERER CMS \& ROS LF. 2009. Heterogeneidades dos reservatórios Flúvio-eólicos da Formação Sergi na Bacia do Recôncavo. Boletim de Geociências da Petrobras, 17: 249-271.
SCHOTS HA. 1990. Tomografia Sísmica Poço a Poço e Poço a Superfície utilizando Ondas Diretas. Master dissertation, Universidade Federal da Bahia, Salvador, Brazil. 73 pp.

SILVA OB, CAIXETA JM, MILHOMEM PS \& KOSIN MD. 2007. Bacia do Recôncavo. Boletim de Geociências da Petrobras, 15: 423-431.

STEWART RR. 1991. Exploration Seismic Tomography: Fundamentals Society of Exploration Geophysicists, Tulsa, OK. 200 pp.

\section{APPENDIX A:}

\section{The Conjugate Gradient Method}

The Conjugate Gradient (CG) is an optimization method to find the solution of a linear system $\boldsymbol{A x}=\boldsymbol{y}$. The CG is an iterative method that starts with an initial estimate of $\boldsymbol{x}_{0}$ that will be refined at each step based on the minimization of the residual $\boldsymbol{r}^{k}=\boldsymbol{y}-\boldsymbol{A} \boldsymbol{x}^{k}$. Because of rounding errors in the numerical calculations, the estimate for $\boldsymbol{x}_{\text {niter }}$ will not be the exact solution but will be a good approximation (Hestenes \& Stiefel, 1952). In this case, the matrix $\boldsymbol{A}$ is symmetrically positive and, up to niter iterations, we will have find a satisfactory approximation for the solution of the system. The following formulas express the CG method:

$$
\begin{aligned}
& \boldsymbol{p}_{0}=\boldsymbol{r}_{0}=\boldsymbol{y}-\boldsymbol{A} \boldsymbol{x}_{0} \quad\left(\boldsymbol{x}_{\mathbf{0}} \text { arbitrary }\right) \\
& \alpha_{i}=\frac{\left(\boldsymbol{p}_{i}, \boldsymbol{r}_{i}\right)}{\left(\boldsymbol{p}_{i}, \boldsymbol{A} \boldsymbol{p}_{i}\right)} \\
& \boldsymbol{x}_{i+1}=\boldsymbol{x}_{i}+\alpha_{i} \boldsymbol{p}_{i} \\
& \boldsymbol{r}_{i+1}=\boldsymbol{r}_{i}-\alpha_{i} \boldsymbol{A} \boldsymbol{p}_{i}, \\
& \beta_{i}=-\frac{\left(\boldsymbol{r}_{i+1}, \boldsymbol{A} \boldsymbol{p}_{i}\right)}{\left(\boldsymbol{p}_{i}, \boldsymbol{A} \boldsymbol{p}_{i}\right)} \\
& \boldsymbol{p}_{i+1}=\boldsymbol{r}_{i+1}+\beta_{i} \boldsymbol{p}_{i} .
\end{aligned}
$$

where $\left(\boldsymbol{p}_{i}, \boldsymbol{r}_{i}\right)$ represents the inner product between vectors $\boldsymbol{p}_{i}$ and $\boldsymbol{r}_{i}$ and so on, $\boldsymbol{p}_{i}$ is the direction that leads to a minimum, and $\alpha$ is the step in the direction of the gradient $\boldsymbol{g}_{i}=-\boldsymbol{r}_{i}$. T0 avoid repeatedly taking the same direction, the term $\boldsymbol{g}_{i}$ is used to correct $\boldsymbol{x}_{i+1}$. The CG method suggests that we take a set of directions $\boldsymbol{p}_{i}$ such that the gradient vector is orthogonal to the new direction.

\section{APPENDIX B:}

\section{Traveltime Seismic Tomography and Ray Tracing}

Tomography is an image reconstruction technique with many applications in Geophysics (Stewart, 1991). It is based on the idea that a set of observed data consist of integrals along lines of some 
physical quantity:

$$
t_{i}=\int_{R_{i}} s(x, z) d l=g[s(x, z)],
$$

where $t_{i}$ is the traveltime related to the $i$-th raypath, $R_{i}$ is the raypath along which the integration is computed, $d l$ is the raypath element, and $s(x, z)$ is the slowness of the block. The middle point is $(x, z)$, where $x$ is the horizontal coordinate and $z$ is the vertical coordinate. $g[s(x, z)]$ represents the variable $g$ as a function of the slowness $s(x, z)$. Equation (B1) has a non-linear behavior because it describes the raypath for non-homogeneous media.

A simpler way to parameterize the velocity distribution is to divide the study area into small cells or blocks and to assign constant values of slowness (reciprocal of velocity) to each cell. The use of high-frequency sources allows for a precise determination of traveltimes, which will provide high-resolution images of velocity structures.

In the geometric acoustics approach, the energy may be transported along curves whose trajectories are orthogonal to the wavefront movement. A more logical way to analyze the ray trace without using the concept of the wavefront is through Fermat's principle. Several methods are described in the literature to determine the ray path between two points. The following steps describe the numerical algorithm proposed by Andersen \& Kak (1982). Applying Fermat's principle and knowing that Euler's equation is a necessary condition for the existence of an extreme value of the integral $\int_{P_{1}}^{P_{2}} n d s$, we obtain the following differential equation for a non-homogeneous medium:

$$
\frac{d}{d s}\left(n \frac{d \boldsymbol{r}}{d s}\right)=\nabla n,
$$

where $n(x, z)$ is the refraction index at position $(x, z), \boldsymbol{r}$ is the ray position vector, $d \boldsymbol{r} / d s$ is a vector tangent to the ray at $(x, z)$, $d s$ is the length element in the ray trajectory, and $\nabla n=d n / d \boldsymbol{r}$ is the refraction index gradient. This differential equation is referred to as the ray equation of the radius, and for a certain regular neighborhood (where the refraction index varies smoothly), its solution represents a family of rays with a shorter acoustic path (Andersen \& Kak, 1982).

Developing the ray equation, we obtain:

$$
\left(\nabla n \cdot \frac{d \boldsymbol{r}}{d s}\right) \frac{d \boldsymbol{r}}{d s}+n \frac{d^{2} \boldsymbol{r}}{d s^{2}}=\nabla n .
$$

Expanding the position vector in the Taylor series at the point $s+\Delta s$, where $\Delta s$ is the ray increment, and considering only the first three terms, we have:

$$
\boldsymbol{r}(s+\Delta s)=\boldsymbol{r}(s)+\frac{d \boldsymbol{r}}{d s} \Delta s+\frac{1}{2} \frac{d^{2} \boldsymbol{r}}{d s^{2}}(\Delta s)^{2} .
$$

Isolating the curvature vector $d^{2} \boldsymbol{r} / d s^{2}$ in Eq. (B3) and substituting it into Eq. (B4), we obtain the following expression:

$$
\begin{aligned}
& \boldsymbol{r}(s+\Delta s)=\boldsymbol{r}(s)+\frac{d \boldsymbol{r}}{d s} \Delta s+\frac{1}{2 n} \\
& \times\left[\nabla n-\left(\nabla n \cdot \frac{d \boldsymbol{r}}{d s}\right) \frac{d \boldsymbol{r}}{d s}\right](\Delta s)^{2} .
\end{aligned}
$$

The next point along the ray is estimated by the following equations:

$$
\begin{gathered}
x_{k+1}=x_{k}+\cos \alpha_{k} \Delta s \\
+\frac{1}{2 s_{k}}\left(s_{k, x}-d_{k} \cos \alpha_{k}\right) \Delta s^{2}, \\
z_{k+1}=z_{k}+\sin \alpha_{k} \Delta s \\
+\frac{1}{2 s_{k}}\left(s_{k, z}-d_{k} \sin \alpha_{k}\right) \Delta s^{2},
\end{gathered}
$$

where $s_{k, x}$ and $s_{k, z}$ are the slownesses in the $x$ and $z$ directions, respectively. $d_{k}$ is defined as:

$$
d_{k}=s_{k, x} \cos \alpha_{k}+s_{k, z} \sin \alpha_{k} .
$$

Starting from a given initial point $\left(x_{0}, z_{0}\right)$ which corresponds to the source position, one may obtain the successive points along the ray because the values of $\sin \alpha_{k}$ and $\cos \alpha_{k}$ are easily calculated. According to Andersen \& Kak (1982), this method has some limitations, as the errors caused in the discretization process or due to the abrupt velocity transitions may be cumulative. To minimize this problem, one must adopt a grid with a sufficient resolution so that the medium is properly sampled, resulting in smoother velocity transitions. One can also smooth the velocity field and use the bilinear interpolation of the refraction index and its partial derivatives.

One problem in ray tracing is finding the coordinates of the receiver where the ray comes from the source. For a homogeneous medium, the connection between the source and the receiver is a straight ray, which makes the problem trivial. Considering a nonhomogeneous medium, however, one must account for the ray curvature. In this case, the problem is predicting the launch angle of the ray from the source so the ray reaches a particular receiver. The bonding process between source and receiver is called ray linking. For this study, the adopted methodology for the ray tracing is based in Schots (1990): the angle and the traveltime for each ray is calculated using the linking method, where the initial angle between source and receiver is a straight line in relation to the horizontal axis. If there is a strong variation in the slowness 
distribution, several rays may not reach the goal after an established number of iterations. In this case, after tracing all rays, one uses as initial angle for non-convergent rays the ray angles that are closer to the rays that have converged. Using the shooting method and considering a circle of a given radius, with center at the receiver $\left(x_{g}, z_{g}\right)$, any ray that enters and finishes in the area will belong to this receiver. The Newton-Raphson method, which compares the traveltime of this ray with the traveltime given from the linking method or of a previous ray, is used to find the receiver. The chosen ray will be the one with the shorter time, according to Fermat's principle.

Recebido em 1 dezembro, 2013 / Aceito em 26 fevereiro, 2015

Received on December 1, 2013 / Accepted on February 26, 2015

\section{NOTES ABOUT THE AUTHORS}

Naiane Pereira de Oliveira holds a B.Sc. degree in Geophysics (2010) and a M.Sc. in Geophysics (2013), both from the Universidade Federal da Bahia. Worked as a geophysicist at GEORADAR in 2012. Currently, is teaching Physics at the Area 1 University. Has experience in Geophysics in the following topics: seismic acquisition, seismic processing, seismic inversion and seismic and electromagnetic tomography.

Amin Bassrei holds a B.Sc. degree in Electrical Engineering (1985) and a D.Sc. degree in Geophysics (1990), both from the Universidade Federal da Bahia (UFBA). Post-doctoral training in Petroleum Geophysics at MIT's Earth Resources Laboratory (1992-1993). Faculty at UFBA since 1995 and is currently associate professor IV at the Department of Geophysics and researcher at the Research Center in Geophysics and Geology (CPGG/UFBA). Undergraduate coordinator (2004-2008) and graduate coordinator (2009-2011, 2013-2015) in the Geophysics Program at UFBA. Since 2009 is coordinator of the CT-PETRO Research Network in Exploration Geophysics - FINEP Rede 01. Experience in exploration seismology, seismic and gravity inversion, seismic tomography, signal analysis, and $\mathrm{CO}_{2}$ geological storage. 